\title{
Kosten und Übergewicht
}

Dr. Bertino Somaini ist Direktor der Gesundheitsförderung Schweiz, einer Stiftung die sich spezifisch für Anliegen der Gesundheitsförderung und die Verbindung zur Prävention einsetzt

Übergewicht nimmt in vielen Ländern stark zu. In dieser Ausgabe gehen Schmid et al. und Neilson et al. auf die dabei entstehenden Folgekrankheiten und deren Kosten ein. Übergewicht verursacht viele Probleme und Krankheiten. Vielfältig ist auch deren medizinische Behandlung, die meistens keine Heilung bringt. Unser medizinisches System investiert heute schon grosse Summen in diese Behandlung und auch in die Entwicklung neuer aufwendiger Behandlungsmethoden. Ein ähnlicher Wille in die Prävention zu investieren ist kaum auszumachen. Die Forderung nach „mehr Prävention“ wird zwar lautstark verkündet, aber nicht nachhaltig gefördert. Prävention und Gesundheitsförderung ist eigentlich der entscheidende und nachhaltige Ansatz, um das Problem Übergewicht und deren Folgekrankheiten zu verringern. Leider hat aber Prävention und Gesundheitsförderung in der Gesellschaft wie auch in der
Politik einen geringen Stellenwert. Solange der so bleibt, werden wir weiterhin interessiert Berichte lesen über die Zunahme von Übergewicht, Forscher werden die Daten noch detaillierter analysieren und mit Studien gewisse Zusammenhänge belegen. Der Nobelpreisträger (Oekonomie) Douglass C. North konnte überzeugend darstellen „Bestehende Systeme lenken das Unternehmertum und die Innovation. Wenn ein staatliches System beispielsweise Piratentum, belohnt', werden Organisationen viel investieren, um die best möglichen Seeräuber auszubilden und Piratenorganisationen werden florieren!“

Was wäre wohl in 30-40 Jahren, wenn unser System ab heute Prävention und Gesundheitsförderung „belohnen“ würde?

Bertino Somaini

\section{| Literaturverzeichnis}

Douglass CN (1990). Institutions, institutional change and economic performance. Cambridge: University Press.

Neilson A, Schneider H (2005). Obesity and its comorbidities: present and future importance on health status in Switzerland. Soz Praventiv Med 50: 78-86.
Schmid A, Schneider H, Golay A, Keller $U$ (2005). Economic burden of obesity and its comorbidities in Switzerland. Soz Praventiv Med 50: 87-94.
Korrespondenzadresse

Dr. Bertino Somaini

Gesundheitsförderung Schweiz

Dufourstrasse 30

Postfach 311

CH-3000 Bern 6

e-mail: bertino.somaini@promotionsante.ch 